\title{
Effects of rotenone on inducible nitric oxide synthase and cyclooxygenase- 2 mRNA levels detected by real-time PCR in a rat bladder ischemia/reperfusion model
}

\author{
IDRIS NERGIZ ${ }^{1}$, BARBAROS BAŞESKIOĞLU ${ }^{1}$, AYDIN YENILMEZ ${ }^{1}$, \\ NILÜFER ERKASAP ${ }^{2}$, CAVIT CAN $^{1}$ and MURAT TOSUN ${ }^{3}$ \\ Departments of ${ }^{1}$ Urology and ${ }^{2}$ Physiology, Eskisehir Osmangazi University Medical Faculty, Eskisehir; \\ ${ }^{3}$ Department of Histology and Embryology, Afyon Kocatepe University Medical Faculty, Afyon, Turkey
}

Received February 3, 2012; Accepted May 14, 2012

DOI: $10.3892 / \mathrm{etm} .2012 .596$

\begin{abstract}
We aimed to determine whether rotenone treatment prevents induced ischemia/reperfusion $(\mathrm{I} / \mathrm{R})$ damage in rat bladders by detecting inducible nitric oxide synthase (iNOS) and cyclooxygenase-2 (COX-2) levels by real-time PCR (RT-PCR). A total of 18 Sprague-Dawley albino rats were used in this experiment. The experimental groups each consisted of 6 rats and were treated as follows: group I, control; group II, I/R; group III, rotenone + I/R. In the control group, the rat bladders were removed by lower abdominal incision without any procedure. In the I/R group, $1 \mathrm{~h}$ prior to the ischemia $1 \mathrm{cc}$ physiological serum was administered and the abdominal aortas were clamped for $1 \mathrm{~h}$ to achieve bladder ischemia. Following the ischemia, reperfusion was induced for $1 \mathrm{~h}$ and the bladders were removed. In the rotenone $+\mathrm{I} / \mathrm{R}$ group, the rats were treated with $25 \mathrm{mg} / \mathrm{kg}$ rotenone intraperitoneally. The iNOS and COX-2 mRNA levels in each group were detected using RT-PCR. In the I/R group, the COX-2 levels in the bladder tissue were higher compared with the control group $(\mathrm{P}<0.05)$. The COX-2 levels in the rotenone-treated group were statistically lower compared with the I/R group $(\mathrm{P}<0.01)$. Vascularization and edema were markedly increased in the I/R group. Following rotenone treatment these were abrogated inversely to inflammation. Although iNOS levels were slightly higher in the I/R group compared with the control group, iNOS levels did not decrease and no significant difference was observed between the groups with regard to
\end{abstract}

Correspondence to: Dr Barbaros Başeskioğlu, Department of Urology, Eskisehir Osmangazi University Hospital, Büyükdere Mah., Meselik, Eskisehir 26450, Turkey

E-mail: barbaroza@gmail.com

Abbreviations: iNOS, inducible nitric oxide synthase; COX-2, cyclooxygenase-2; RT-PCR, real-time polymerase chain reaction

Key words: rotenone, bladder, rat, ischemia/reperfusion, inducible nitric oxide synthase, cyclooxygenase- 2 rotenone treatment $(\mathrm{P}>0.05)$. We suggest that rotenone may be used clinically to treat $\mathrm{I} / \mathrm{R}$ damage due to its diminishing effect on COX-2 levels.

\section{Introduction}

A lack of blood supply to the tissues is termed ischemia and leads to cell death and organ failure. The lesions become more severe with the supply of blood in the reperfusion phase (1). Ischemia/reperfusion (I/R) injury is a series of cellular events triggered by the inadequate supply of oxygen $\left(\mathrm{O}_{2}\right)$ to the tissues, and ultimately all organs and systems are affected. Reperfusion and reoxygenation of the ischemic tissue causes lipid peroxidation of cell membranes and produces free oxygen radicals (FORs) that lead to alterations in cellular functions $(2,3)$. FORs, which are produced due to the reperfusion of ischemic tissue, have been found to be responsible for this damage (4). It has been shown that catheterization following the overdistension of the urinary bladder initiates reperfusion injury and that FORs are one of the main factors in this damage $(5,6)$. Previous studies have shown that FORs and peroxynitrite, produced due to I/R injury, cause urinary bladder dysfunction by means of affecting bladder components, including smooth muscle, mucosa and peripheral nerves (5).

Bladder ischemia occurs as a result of a reduction in the blood supply caused by atherosclerosis or trauma. This leads to impairment in compliance during the contraction of the bladder and may consequently result in bladder dysfunction. It has been shown that an increase in bladder wall thickness mediates a cyclic I/R in each micturition (7). Bladder dysfunction observed following decompression or catheterization, which is performed following an acute overdistension or retention, is caused by $\mathrm{I} / \mathrm{R}(5,8)$. Cyclooxygenase (COX) isoforms (COX-1 and COX-2) catalyze the initial step of arachidonic acid metabolism. COX-2 expression is increased in a number of organs during I/R injury. Previous studies have reported that COX-2 inhibitors may protect the tissue from I/R injury (9). Nitric oxide (NO) is a significant endogenous regulatory molecule that plays a role in various biological functions and is involved in the regulation of homeostasis. Previous studies 
have highlighted that NO levels were increased in the damage which occurred after bladder I/R injury and that the therapies which aimed to reduce NO levels were successful in preventing this damage (10). Complex I is the first enzyme complex in the electron transport chain of mitochondria and is a significant source for the production of reactive oxygen species. Rotenone, a mitochondrial electron transport inhibitor, takes part in the anti-oxidative defense mechanism (11).

In this present study, we aimed to ascertain whether rotenone treatment inhibits experimentally induced I/R injury in the rat urinary bladder, by means of measuring COX-2 and inducible nitric oxide synthase (iNOS) mRNA levels.

\section{Materials and methods}

Animals. This experimental study was conducted following approval of the ethics committee (date, 26.03.2008; number $44 / 2208)$. Sprague Dawley rats of both genders, aged 5-6 months and weighing between 250 and $300 \mathrm{~g}$ were used for the study. The rats were divided into 3 groups and 18 rats, 6 in each group, were used for real-time polymerase chain reaction (RT-PCR) assays. The rats were anesthetized by intraperitoneal (i.p.) injection of $50 \mathrm{mg} / \mathrm{kg}$ ketamine with $20 \mathrm{mg} / \mathrm{kg}$ rompun. Following the anesthesia, median abdominal laparotomy was performed.

Group I (control group, $n=6$ ). The urinary bladders of the rats were removed without any intervention immediately after performing abdominal laparotomy.

Group II (I/R group, $n=6)$. The rats in this group were administered normal saline $1.0 \mathrm{cc}$ i.p. $1 \mathrm{~h}$ before the ischemia, and bladder ischemia was induced by clamping the abdominal aorta for $1 \mathrm{~h}$ with a non-traumatic microvascular clamp (bulldog clamp). During this process, the laparotomy incision was sutured with $3 / 0$ silk. After the ischemia period, the laparotomy incision was reopened, the abdominal aorta was unclamped and in order to provide reperfusion for $1 \mathrm{~h}$, the laparotomy incision was re-sutured and closed. After 1-h reperfusion, the bladder tissues were removed.

Group III (rotenone + IR group, $n=6$ ). The rats in this group were administered rotenone i.p. at a dose of $25 \mathrm{mg} / \mathrm{kg} 1 \mathrm{~h}$ prior to ischemia induction by clamping the abdominal aorta. Thereafter, laparotomy was performed through an abdominal incision and bladder ischemia was induced by clamping the abdominal aorta for $1 \mathrm{~h}$ with a non-traumatic microvascular clamp. The laparotomy incision was closed by suturing with $3 / 0$ silk. After the ischemia period, the laparotomy incision was re-opened, the clamp was removed and reperfusion was provided for $1 \mathrm{~h}$ and then the laparotomy incision was re-sutured and closed. Following 1-h reperfusion, the bladder tissues were removed.

Histology. The specimens were fixed in $10 \%$ neutral formalin, dehydrated in increasing alcohol series, cleared in xylene and embedded in paraffin. Several $4-\mu \mathrm{m}$ sections obtained from these specimens were mounted onto slides. All specimens were stained with hematoxylin and eosin (H\&E) for histochemistry and evaluated under light microscopy.
RNA extraction and RT-PCR. The mRNA levels of iNOS and COX-2 in relation to the housekeeping gene glyceraldehyde 3-phosphate dehydrogenase (GAPDH) were determined using RT-PCR with SYBR-Green. Total RNA was extracted from urinary bladder tissue using an RNA stabilization reagent (Qiagen, Valencia, CA, USA), according to the manufacturer's instructions, and quantified by measuring the absorbance at 260 nm (Nanodrop 1000; Thermo, Wilmington, DE, USA). Aliquots of $20 \mu \mathrm{l}$ of RNA from each group were used to produce complementary DNA (cDNA). The newly synthesized cDNA, stored at $-20^{\circ} \mathrm{C}$, was used for the mRNA assay of iNOS and COX-2 with RT-PCR. cDNA $(1 \mu \mathrm{l})$ from each group was amplified in $25 \mu \mathrm{l}$ of reactive mixture with $0.25 \mathrm{X}$ SYBR-Green Supermix (Molecular Probes, Invitrogen, Carlsbad, CA, USA). RT-PCR was performed by monitoring the increase in the amount of SYBR-Green in real time using Rotor-Gene 6000 RT-PCR (Corbett Research, Sydney, Australia). The oligonucleotide sequences of the cDNA primers were designed at Gene Research Laboratories, UK. The forward primer for rat iNOS was 5'-CACCACCCTCCTTGTTCAAC-3' and the reverse primer was 5'-CAATCCACAACTCGCTCCAA-3'. The forward primer for rat COX-2 was 5'-TGCGATGCTCTT CCGAGCTGTGCT-3' and the reverse primer was 5'-TCA GGAAGTTCCTTATTTCCTTTC-3'. Sobajima et al also used GAPDH (housekeeping gene) to normalize iNOS (target gene) data using RT-PCR (12).

The RT-PCR thermal cycling conditions were as follows: $5 \mathrm{~min}$ at $65^{\circ} \mathrm{C}, 60 \mathrm{~min}$ at $37^{\circ} \mathrm{C}$ for cDNA synthesis, $15 \mathrm{~min}$ at $95^{\circ} \mathrm{C}, 15 \mathrm{sec}$ at $95^{\circ} \mathrm{C}, 1 \mathrm{~min}$ at $60^{\circ} \mathrm{C}$ for 50 cycles and $1 \mathrm{~min}$ at $55^{\circ} \mathrm{C}$. RT-PCR data were collected using the Rotor-Gene 6000 detection system. Cycle threshold (CT) values were determined by automated threshold analysis. The primer quality (lack of primer-dimer amplification) was confirmed by melting curve analysis. The relative quantification of the gene expression was performed using the standard curve method, constructed with serial dilutions of control mRNA or RT-PCR amplicons. All experiments were carried out in triplicate. iNOS and COX-2 levels were standardized to GAPDH (ratio iNOS:GAPDH) to account for loading differences. Gene expression levels (mRNA) were reported using the median as a point estimator and the range of values.

Statistical analysis. SPSS (Statistical Package for Social Sciences, Inc., Chicago, IL, USA) v. 10.0 package program was used for the statistical evaluation. Statistical significance between the groups was analyzed using the Tukey's HSD multiple comparison test. The data are presented as mean and standard deviation (mean $\pm \mathrm{SD}$ ). $\mathrm{P}<0.05$ was considered to indicate a statistically significant result.

\section{Results}

Histological results. The specimens were evaluated under light microscopy and inflammation criteria, including edema, vascularization and infiltration of immune cells, were scored. Severe criteria were described as +++ , mild criteria as ++ and slight criteria as + . During the evaluation of the slides it was noted that vascularization and edema were markedly increased in the $\mathrm{I} / \mathrm{R}$ group. However, following rotenone treatment these were decreased to lower degrees. On the other 
A

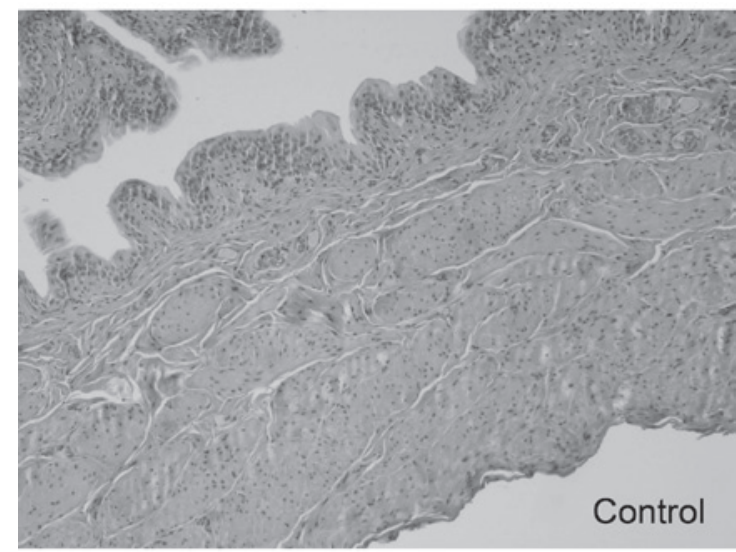

B

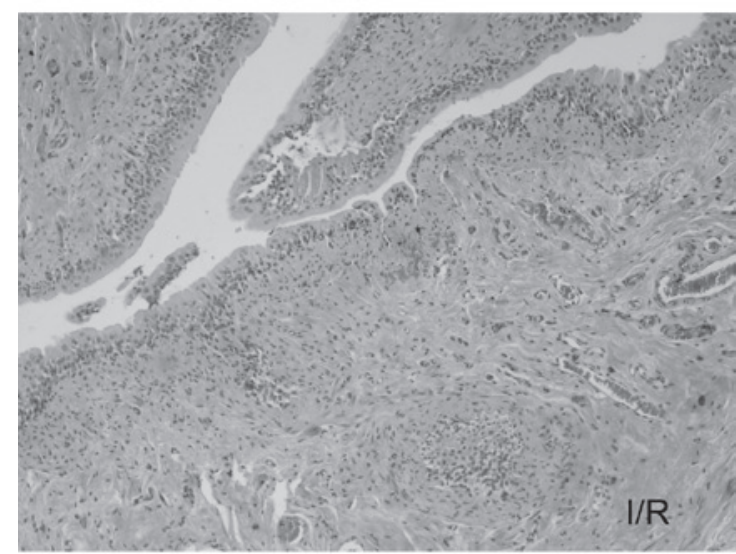

C

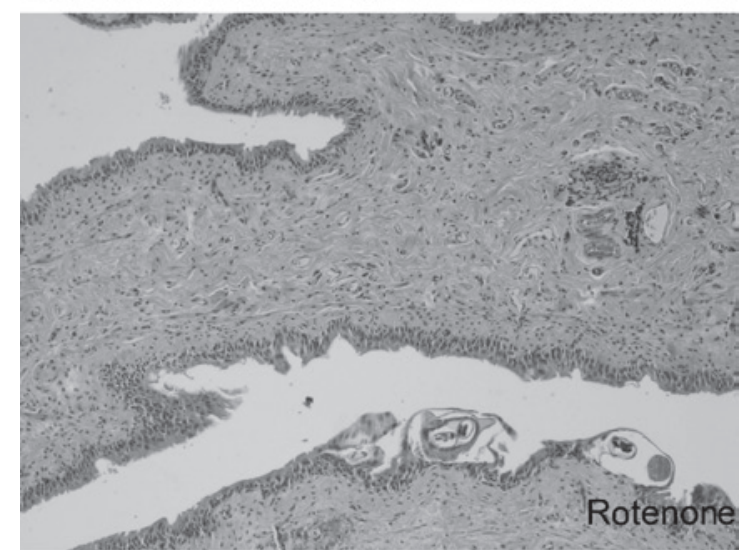

Figure 1. (A) Control. (B) Vascularization, inflammation and edema were markedly increased in the I/R group. (C) Following rotenone treatment, vascularization and edema were significantly reduced with a small decrease in inflammation. I/R, ischemia/reperfusion.

hand, inflammation in the $\mathrm{I} / \mathrm{R}$ group was markedly higher than in the control group. Following rotenone treatment, it was slightly decreased. All the scores are summarized in Table I. $\mathrm{H} \& \mathrm{E}$ staining of all the structures in the bladder are shown in Fig. 1.

Expression of iNOS in urinary bladder. iNOS levels were increased in the I/R group and iNOS values were not decreased with rotenone administration prior to $\mathrm{I} / \mathrm{R}$. According to one-way analysis of variance, the three groups were not significantly different $(\mathrm{P}=0.194)$. The three groups were also not different when the groups were compared with each other (all P>0.05 by the Tukey test; Fig. 2).
Table I. H\&E-stained sections of the bladder samples.

\begin{tabular}{lccc}
\hline Group & Inflammation & Edema & Vascularization \\
\hline Control & + & - & + \\
I/R & +++ & ++ & +++ \\
Rotenone & ++ & + & ++ \\
\hline
\end{tabular}

$\mathrm{H} \& \mathrm{E}$, hematoxylin and eosin; I/R, ischemia/reperfusion. + , slight; ++ , mild; +++ , severe.

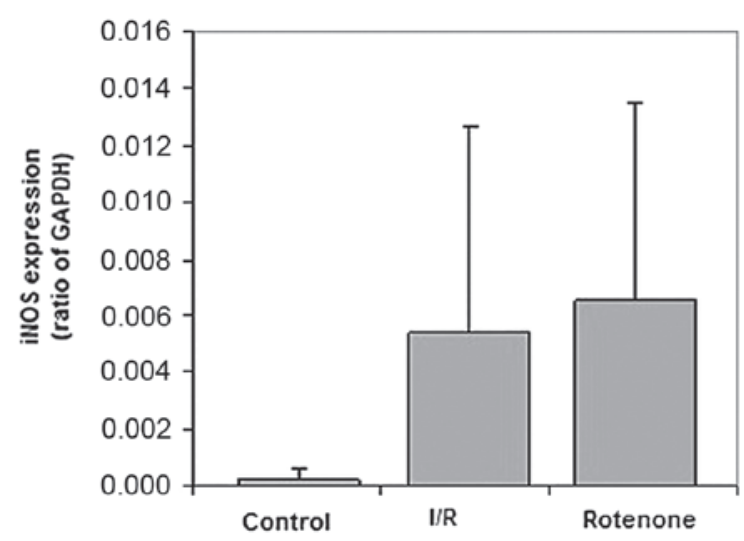

Figure 2. Effect of rotenone on bladder tissue iNOS levels. I/R, ischemia/ reperfusion; iNOS, inducible nitric oxide synthase.

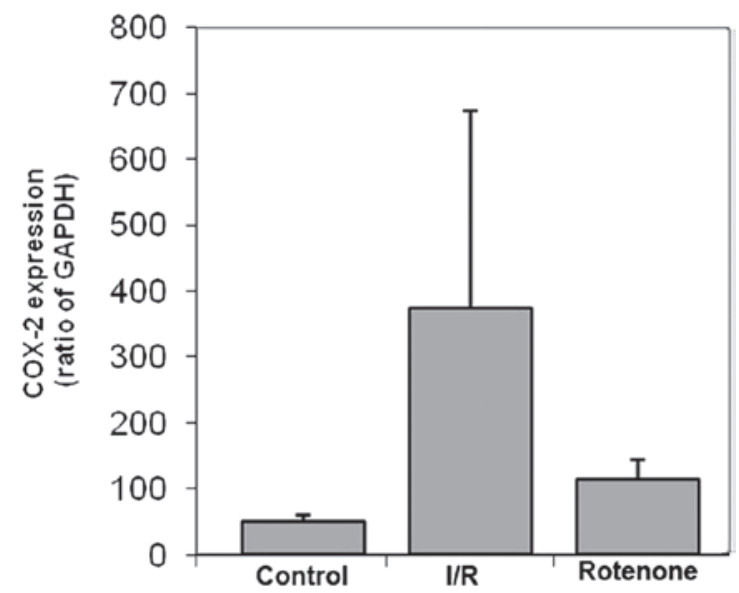

Figure 3. Effect of rotenone on bladder tissue COX-2 levels. I/R, ischemia/ reperfusion; COX-2, cyclooxygenase-2.

Expression of COX-2 in urinary bladder. I/R significantly increased the COX-2 values $(\mathrm{P}<0.05)$ and $C O X-2$ values were not increased with rotenone administration prior to $I / R$, but were decreased to below the values noted in the control group (P<0.01; Fig. 3).

\section{Discussion}

An increase in FORs following I/R causes bladder tissue damage (5). In the present study, rotenone treatment prevented bladder damage via the COX-2 pathway. This hypothesis was verified in the histological examination which revealed that 
inflammation, edema and vascularization were higher in the I/R group and that rotenone treatment reduced these damaging effects. In this present study, the analysis of the iNOS gene (target gene) expression using the RT-PCR method revealed that the extremely low, almost immeasurable iNOS gene expression was increased following stimulation, such as I/R injury. However, when the relative concentrations (the ratio to the reference GAPDH gene) were taken into consideration, we found that the increment in the I/R group was not statistically significant $(\mathrm{P}>0.05)$. We observed that iNOS expression was not decreased with rotenone treatment as compared with the $\mathrm{I} / \mathrm{R}$ group $(\mathrm{P}>0.05)$.

Urinary bladder ischemia followed by reperfusion may also be observed in age-related conditions, including urinary retention, atherosclerosis, vasospasms, embolism and thrombosis. The development of I/R secondary to these conditions may lead to urinary bladder dysfunction (13). It has been demonstrated that bladder wall blood flow (ischemia) and oxygen pressure (hypoxia) are decreased in partial bladder outlet obstruction and that the increase in the bladder wall thickness leads to cyclic I/R during each micturition (14). However, it has been reported that decompression following acute over-distension or retention and bladder dysfunction observed following catheterization were also associated with I/R $(5,6)$. Saito and Miyagawa reported that histopathological changes, including severe erythrocyte extravasation and leukocyte infiltration, resulted from the rupture of microcirculation in the bladder. I/R injury occurred particularly in the smooth muscles and submucosa, but not in the mucosa, and these histopathological alterations associated with I/R injury were prevented by $N(G)$ nitro-L-arginine methyl ester (L-NAME) treatment (8).

Rotenone has been used as an antioxidant agent in the I/R injury of various organs. Ichikawa et al evaluated I/R in the intestinal mucosa and biochemical analyses revealed that the products that caused the damage were significantly decreased in the group receiving rotenone therapy compared with the ischemia group. The authors stated that rotenone induced this positive effect by inhibiting lipid peroxidation and decreasing mucosal inflammation (15). Vanden Hoek et al attempted to reduce the oxidative stress-induced damage on cardiomyocytes with complex 1 inhibitors and used rotenone, cyanide and antimycin for this purpose; consequently, the authors showed that rotenone was more effective than the other agents (16). Viñas et al reported that, according to quantitative analysis, $\mathrm{I} / \mathrm{R}$ increased the renal expression of iNOS compared with the control and that no differences were found between I/R and the various selective NOS inhibitors (1). In the present study, quantitative iNOS expression increased in the I/R group and no significant difference was observed between the I/R and rotenone treatment groups, similar to the study performed by Viñas et al.

Similarly, we observed that urinary bladder I/R injury in rats was decreased with rotenone treatment. To the best of our knowledge, the expression levels of COX-2 and iNOS have been studied by RT-PCR to detect the I/R injury in various organs, but not in the urinary bladder. In the experimental unilateral lower extremity I/R injury model, Dupouy et al investigated the levels of COX-1 and COX-2 and reported that COX-2 mRNA levels in the skeletal muscle were significantly increased in the I/R group compared with the control group, and that COX-2 inhibitors may protect the tissue against this damage (9). In experimental gastric I/R injury, Hiratsuka et al reported that the COX-2 levels in mesenchymal cells was significantly increased in the I/R group compared with the control group (17). In their study on renal I/R injury, Matsuyama et al suggested that COX-2 played a significant role in organ and tissue homeostasis and that COX-2 inhibitor use may be beneficial in renal I/R injury (18). Tetsuka et al stated that rotenone did not inhibit IL-1 $\beta$-induced Cox- 2 mRNA and iNOS mRNA expression but inhibited IL-1 $\beta$-induced Cox-2 and iNOS protein expression. These data suggest that rotenone inhibited the Cox-2 and iNOS expression at a post-transcriptional level (19). When the relative concentration of COX-2 mRNA (the ratio to the reference GAPDH gene) was evaluated, we also observed that COX-2 mRNA was increased in I/R injury, while significantly decreased following rotenone treatment $(\mathrm{P}<0.01)$.

In conclusion, we observed that rotenone therapy may be beneficial in decreasing the oxidative stress-induced COX-2 levels in urinary bladder I/R injury in rats. However, further studies are required for rotenone therapy to be used for therapeutic purposes in the I/R damage induced in the urinary bladder by various conditions.

\section{References}

1. Viñas JL, Sola A, Genescà M, Alfaro V, Pí F and Hotter G: NO and NOS isoforms in the development of apoptosis in renal ischemia/ reperfusion. Free Radic Biol Med 40: 992-1003, 2006.

2. Levin RM, Leggett R, Whitbeck $C$ and Horan P: Effect of calcium and calcium chelator on the response of the bladder to in vitro ischaemia. Br J Urol 82: 882-887, 1998.

3. Ohnishi N, Liu SP, Horan P and Levin RM: Effect of repetitive stimulation on the contractile response of rabbit urinary bladder subjected to in vitro hypoxia or in vitro ischemia followed by reoxygenation. Pharmacology 57: 139-147, 1998.

4. Wang JH, Chen HS, Wang T, Tian KL and Diao YF: Role of oxygen-derived free radicals in superior mesenteric artery occlusion shock in rats. Chin Med J (Engl) 103: 278-282, 1990.

5. Lin AT, Chen KK, Yang CH and Chang LS: Mannitol facilitates rabbit urinary bladder recovery from overdistension injury. Urology 56: 702-707, 2000.

6. Saito M and Miyagawa I: Bladder dysfunction after acute urinary retention in rats. J Urol 165: 1745-1747, 2001.

7. Greenland JE and Brading AF: Urinary bladder blood flow changes during the micturition cycle in a conscious pig model. $\mathrm{J}$ Urol 156: 1858-1861, 1996.

8. Saito $\mathrm{M}$ and Miyagawa I: N(G)-nitro-L-arginine methylester, a nitric oxide synthase inhibitor, diminishes apoptosis induced by ischemia-reperfusion in the rat bladder. Neurourol Urodyn 21: 566-571, 2002.

9. Dupouy VM, Ferre PJ, Uro-Coste E and Lefebvre HP: Time course of COX-1 and COX-2 expression during ischemia-reperfusion in rat skeletal muscle. J Appl Physiol 100: 233-239, 2006.

10. Conners W, Whitebeck C, Chicester P, Legget L, Lin AD, Johnson A, Kogan B, Levin R and Mannikarottu A: L-NAME, a nitric oxide synthase inhibitor, diminishes oxidative damage in urinary bladder partial outlet obstruction. Am J Physiol Renal Physiol 290: 357-363, 2006.

11. Feldkamp T, Kribben A, Roeser NF, Senter RA, Kemner S, Venkatachalam MA, Nissim I and Weinberg JM: Preservation of complex I function during hypoxia-reoxygenation-induced mitochondrial injury in proximal tubules. Am J Physiol Renal Physiol 286: F749-F759, 2004.

12. Sobajima S, Shimer AL, Chadderdon RC, Kompel JF, Kim JS, Gilbertson LG and Kang JD: Quantitative analysis of gene expression in a rabbit model of intervertebral disc degeneration by real-time polymerase chain reaction. Spine J 5: 14-23, 2005.

13. Gill HS, Monson FC, Wein AJ, Ruggieri MR and Levin RM: The effects of short term in-vivo ischemia on the contractile function of the rabbit urinary bladder. J Urol 139: 1350-1354, 1988. 
14. Bratslavsky G, Kogan BA, Matsumoto S, Aslan AR and Levin RM: Reperfusion injury of the rat bladder is worse than ischemia. J Urol 170: 2086-2090, 2003.

15. Ichikawa H, Takagi T, Uchiyama K, Higashihara H, Katada K, Isozaki Y, Naito Y, Yoshida N and Yoshikawa T: Rotenone, a mitochondrial electron transport inhibitor, ameliorates ischemiareperfusion-induced intestinal mucosal damage in rats. Redox Rep 9: 313-316, 2004.

16. Vanden Hoek TL, Shao Z, Li C, Schumacker PT and Becker LB: Mitochondrial electron transport can become a significant source of oxidative injury in cardiomyocytes. J Mol Cell Cardiol 29: 2441-2450, 1997.
17. Hiratsuka T, Futagami S, Tatsuguchi A, et al: COX-1 and COX-2 conversely promote and suppress ischemia-reperfusion gastric injury in mice. Scand J Gastroenterol 40: 903-913, 2005.

18. Matsuyama M, Nakatani T, Hase T, et al: The expression of cyclooxygenases and lipoxygenases in renal ischemia-reperfusion injury. Transplant Proc 36: 1939-1942, 2004.

19. Tetsuka T, Baier LD and Morrison AR: Antioxidants inhibit interleukin-1-induced cyclooxygenase and nitric-oxide synthase expression in rat mesangial cells. Evidence for posttranscriptional regulation. J Biol Chem 271: 11689-11693, 1996. 